\title{
Strange-hadron correlation studies to investigate strangeness enhancement in pp collisions
}

\author{
Chiara De Martin ${ }^{a, b, *}$ on behalf of the ALICE collaboration \\ ${ }^{a}$ Department of Physics, University of Trieste, \\ Via Valerio, 2, Trieste, Italy \\ ${ }^{b}$ INFN Trieste, \\ via Valerio, 2, Trieste, Italy \\ E-mail: chiara.de.martin@cern.ch
}

The production of strange and multi-strange hadrons in heavy-ion collisions is enhanced with respect to minimum bias proton-proton (pp) collisions. This feature has been further investigated by studying pp collisions as a function of the produced charged-particle multiplicity. In pp collisions, the strange hadron yields normalised to the pion yield show an increase with the multiplicity of produced particles. The origin of this striking phenomenon remains an open question: is it related to soft particle production or to hard scattering events, such as jets?

The ALICE experiment has further studied this feature by separating strange hadrons produced in jets from those produced in soft processes. For this purpose, the angular correlation between high transverse momentum $\left(p_{\mathrm{T}}\right)$ charged particles and strange hadrons has been exploited. In this poster, the recent measurement of the near-side jet yield and the out-of-jet yield of $\mathrm{K}_{\mathrm{S}}^{0}$ and $\Xi$ is shown as a function of the multiplicity of charged particles produced in pp collisions at $\sqrt{s}=13 \mathrm{TeV}$. The ratio between the $\Xi$ and the $\mathrm{K}_{\mathrm{S}}^{0}$ yields is also shown, to highlight the effect of the different strangeness content of the two hadrons.

The results suggest that soft (out of jet) processes are the dominant contribution to strange particle production.

The Ninth Annual Conference on Large Hadron Collider Physics - LHCP2021

7-12 June 2021

Online

${ }^{*}$ Speaker 


\section{Introduction}

Heavy-ion collisions are a unique tool to study the quark-gluon plasma (QGP) [1], a state of matter in which quarks and gluons are not bound within hadrons by the strong force. QGP is expected to form when high energy density and temperature conditions are reached, as in heavy-ion collisions. One proposed signature of QGP formation is the strangeness enhancement effect [2], which consists in an increase of the ratio of strange to non-strange hadron yields in $\mathrm{Pb}$ - $\mathrm{Pb}$ collisions with respect to minimum bias pp collisions. This effect has been further investigated by studying its dependence on the multiplicity of charged particles produced in the collisions [3-7]. Results show that the ratios of different strange hadron yields to pion yields increase with the multiplicity of charged particles, revealing a smooth transition between different collision systems, from low multiplicity pp collisions to high multiplicity central $\mathrm{Pb}-\mathrm{Pb}$ collisions. This behaviour is striking as different particle production mechanisms are expected to be involved in the different collision systems. The strangeness enhancement observed in pp collisions can be qualitatively reproduced by models like PYTHIA [8] and EPOS 3 [9], which are based on very different assumptions on the underlying physics: PYTHIA can reproduce the trend if colour ropes are introduced, while EPOS 3 can explain the trend by changing the core-to-corona ratio with multiplicity. Further studies are therefore needed to shed light on the origin of this phenomenon in small collision systems.

In these proceedings, the production of $\mathrm{K}_{\mathrm{S}}^{0}$ and $\Xi^{ \pm}$in jets and out of jets in pp collisions at $\sqrt{s}=13 \mathrm{TeV}$ with the ALICE detector is discussed. The near-side jet and out-of-jet productions are studied exploiting the correlation between the direction of high- $p_{\mathrm{T}}$ charged particles and the one of strange hadrons. The results provide new insight into the contribution of hard scattering events and of soft particle production processes to the strangeness enhancement effect in small collision systems.

\section{Analysis strategy}

With the purpose of separating strange hadrons produced in jets from those produced out of jets, the angular correlation between high- $p_{\mathrm{T}}$ charged tracks (trigger particles) and strange hadrons is exploited. Strange hadrons are identified with the ALICE detector [10] by exploiting their weak decay topology into charged hadrons: $\mathrm{K}_{\mathrm{S}}^{0}$ are identified via the decay $\mathrm{K}_{\mathrm{S}}^{0} \rightarrow \pi^{+} \pi^{-}$, while $\Xi^{ \pm}$via the decay $\Xi^{-} \rightarrow \Lambda \pi^{-} \rightarrow \mathrm{p} \pi^{-} \pi^{-}$, together with its charge conjugate. The decay products are reconstructed and identified using the Time Projection Chamber (TPC) [11] of the ALICE detector and topological and kinematic selections are applied to reduce the combinatorial background. $\mathrm{K}_{\mathrm{S}}^{0}$ and $\Xi$ are then identified using invariant-mass analysis techniques.

The trigger particle is defined as the highest- $p_{\mathrm{T}}$ charged particle found in the event and satisfying the kinematic selection $p_{\mathrm{T}}>3 \mathrm{GeV} / c$. Trigger particles are considered a proxy for the jet axis. Strange hadrons produced in jets are expected to be found at a small angular distance from the trigger particles, and therefore the angular-correlation distribution in a $(\Delta \eta, \Delta \varphi)$ region centred at $(0,0)$ is used to extract the near-side jet yield, after proper subtraction of the out-of-jet contribution. The out-of-jet contribution is evaluated at larger values of $\Delta \eta$ and $\Delta \varphi$, where no contribution from jet production is expected. The whole angular-correlation distribution is used to obtain the full production yield. 
The analysis is performed in multiplicity classes based on the distribution of the signal amplitude measured in the two V0 detectors placed at forward rapidity [12]. For each multiplicity class an average value of charged particles produced at midrapidity $\langle\mathrm{dN} / \mathrm{d} \eta\rangle_{|\eta|<0.5}$ is assigned.

\section{Results}

The full, out-of-jet and near-side jet $p_{\mathrm{T}}$ spectra of the $\Xi$ baryon in the different multiplicity classes are shown in the left, central and right panels of Figure 1, respectively. As expected, the near-side jet spectra (right panel) are harder than the out-of-jet spectra (central panel), with the latter being related to soft particle production. The same is observed for the $\mathrm{K}_{\mathrm{S}}^{0}$ meson. The $p_{\mathrm{T}}$ spectra are interpolated in order to extract the yield in the $p_{\mathrm{T}}$ intervals were it cannot be measured.
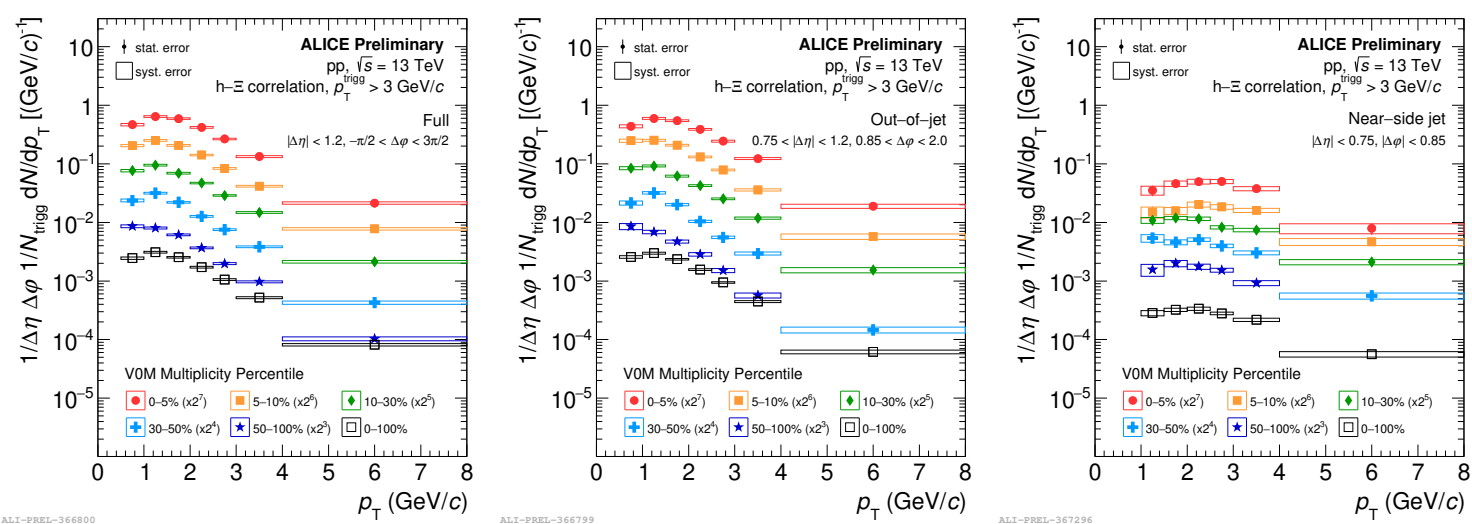

Figure 1: $\Xi p_{\mathrm{T}}$ spectra for full (left), out-of-jet (centre) and near-side jet (right) production. The statistical errors are represented by the error bars and the total systematic uncertainty by the empty boxes.

The left (right) panel of Figure 2 shows the $K_{S}^{0}(\Xi)$ yields per trigger particle normalised to the corresponding $\Delta \eta \Delta \varphi$ area as a function of $\langle\mathrm{dN} / \mathrm{d} \eta\rangle_{|\eta|<0.5}$. For both $\mathrm{K}_{\mathrm{S}}^{0}$ and $\Xi$, the full and out-of-jet yields increase with the multiplicity of charged particles, while the near-side jet yield shows a much milder evolution, suggesting that the relative contribution of soft processes increases with the multiplicity.

The strangeness enhancement effect can be studied by looking at the $\Xi / \mathrm{K}_{\mathrm{S}}^{0}$ yield ratio as a function of $\langle\mathrm{dN} / \mathrm{d} \eta\rangle_{|\eta|<0.5}$ (Figure 3). The increase with multiplicity of the ratio of full yields is attributed to the larger strangeness content of the $\Xi(|S|=2)$ with respect to the $\mathrm{K}_{\mathrm{S}}^{0}(|S|=1)$. The outof-jet ratio increases with multiplicity in a similar way, while firm conclusions about the multiplicity dependence of the near-side jet ratio cannot be drawn due to the large uncertainties. While the out-of-jet ratio is compatible with the ratio of full yields, the near-side jet ratio is smaller for all values of multiplicity, suggesting that soft particle production represents the dominant contribution to the strangeness enhancement effect in pp collisions. 

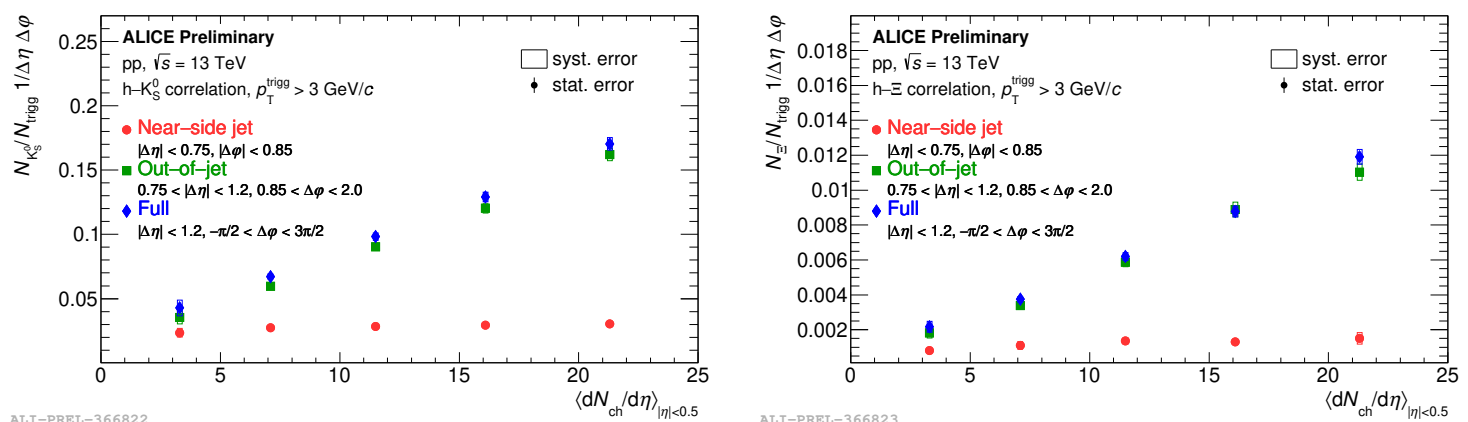

Figure 2: $\mathrm{K}_{\mathrm{S}}^{0}$ (left) and $\Xi$ (right) yields per trigger particle and per unit $\Delta \eta \Delta \varphi$ area vs charged-particle multiplicity at midrapidity $\langle\mathrm{dN} / \mathrm{d} \eta\rangle_{|\eta|<0.5}$ for near-side jet (red markers), out-of-jet (green markers) and full (blue markers) production. The statistical errors are represented by the error bars and the total systematic uncertainty by the empty boxes.



Figure 3: $\Xi / \mathrm{K}_{\mathrm{S}}^{0}$ yield ratio vs charged-particle multiplicity at midrapidity $\langle\mathrm{dN} / \mathrm{d} \eta\rangle_{|\eta|<0.5}$ for near-side jet (red markers), out-of-jet (green markers) and full (blue markers) production. The statistical errors are represented by the error bars and the total systematic uncertainty by the empty boxes.

\section{Conclusions}

The ALICE collaboration has carried out several multi-differential analyses to investigate the origin of the strangeness enhancement effect in small collision systems. In these proceedings, the near-side jet and out-of-jet production of $\mathrm{K}_{\mathrm{S}}^{0}$ and $\Xi$ hadrons was presented as a function of the multiplicity of charged particles produced in pp collisions at $\sqrt{s}=13 \mathrm{TeV}$. Results suggest that soft processes represent the dominant contribution to strange hadron production. 


\section{References}

[1] R. Pasechnik and M. Šumbera, Phenomenological Review on Quark-Gluon Plasma: Concepts vs. Observations, Universe 3 (2017) 7 [1611.01533].

[2] J. Rafelski and B. Müller, Strangeness production in the quark-gluon plasma, Phys. Rev. Lett. 48 (1982) 1066.

[3] ALICE collaboration, Enhanced production of multi-strange hadrons in high-multiplicity proton-proton collisions, Nature Phys. 13 (2017) 535 [1606. 07424].

[4] ALICE collaboration, Multiplicity dependence of (multi-)strange hadron production in proton-proton collisions at $\sqrt{s}=13 \mathrm{TeV}$, Eur. Phys. J. C 80 (2020) 167 [1908. 01861].

[5] ALICE collaboration, Multi-strange baryon production in $p$-Pb collisions at $\sqrt{s_{\mathrm{NN}}}=5.02$ TeV, Phys. Lett. B758 (2016) 389 [1512 . 07227].

[6] ALICE collaboration, Multiplicity Dependence of Pion, Kaon, Proton and Lambda Production in p-Pb Collisions at $\sqrt{s_{N N}}=5.02$ TeV, Phys. Lett. B 728 (2014) 25 [1307.6796].

[7] ALICE collaboration, Multi-strange baryon production at mid-rapidity in $\mathrm{Pb}-\mathrm{Pb}$ collisions at $\sqrt{s_{N N}}=2.76 \mathrm{TeV}$, Phys. Lett. B 728 (2014) 216 [1307. 5543].

[8] T. Sjostrand, S. Mrenna and P.Z. Skands, A Brief Introduction to PYTHIA 8.1, Comput. Phys. Commun. 178 (2008) 852 [0710. 3820].

[9] K. Werner, B. Guiot, I. Karpenko and T. Pierog, Analysing radial flow features in $p-P b$ and p-p collisions at several TeV by studying identified particle production in EPOS3, Phys. Rev. C 89 (2014) 064903 [1312 . 1233].

[10] ALICE collaboration, Performance of the ALICE Experiment at the CERN LHC, Int. J. Mod. Phys. A29 (2014) 1430044 [1402 . 4476].

[11] J. Alme et al., The ALICE TPC, a large 3-dimensional tracking device with fast readout for ultra-high multiplicity events, Nucl. Instrum. Meth. A 622 (2010) 316 [1001. 1950].

[12] ALICE collaboration, Performance of the ALICE VZERO system, Journal of Instrumentation 8 (2013) P10016. 